\title{
Malnutrition in Older Adults - Urgent Need for Action: A Plea for Improving the Nutritional Situation of Older Adults
}

\author{
Dorothee Volkert
}

Institute for Biomedicine of Aging, Friedrich-Alexander-Universität Erlangen-Nürnberg, Nuremberg, Germany

\section{Key Words}

Screening for malnutrition • Geriatric assessment •

Nutritional management · Anorexia of aging

\begin{abstract}
During the past decades, malnutrition has attracted increasing scientific attention and is by now regarded as a true geriatric syndrome characterized by multifactorial causality, identified by symptoms and accompanied by frailty, disability and poor outcome. This viewpoint summarizes our present knowledge and the usual current handling of malnutrition in older people and highlights the urgent need for action in this field. Age-related changes in the complex system of appetite regulation, resulting in the so-called anorexia of aging, predispose older adults to a decrease in food intake which may lead to malnutrition, if additional risk factors like health or social problems occur. Consequently, malnutrition is widespread in the older population, notably in those who are institutionalized. Despite the fact that effective interventions are available, prevention and treatment of malnutrition do not currently receive appropriate attention. As an important first step towards better awareness, screening for malnutrition should become a mandatory integral part of the comprehensive geriatric assessment. Furthermore, practical local guidelines should be implemented in all geriatric hospital wards and nursing homes in order to improve nutritional care in the daily routine. Important to
\end{abstract}

note is that reasonable nutritional management is not possible without qualified staff in adequate numbers allowing appropriate individual nutritional care. Regarding future research, studies at the cellular, metabolic and clinical levels and the linking of information from different research approaches are required to better understand the transition from good nutritional health and independence of old people to malnutrition, functional impairment and poor health. In parallel to well-designed observational and intervention studies, standardized documentation of nutritional information in daily routine would enable the uniform collection of data for research as well as for political decisions. In summary, the time is ripe for better inclusion of nutrition in geriatric health care. This will not only bring about improved nutritional status and outcome, and thus individual benefit for the affected person, but also economic benefits both for the institution and the health-care system.

Copyright $\odot 2013$ S. Karger AG, Basel

\section{Introduction}

Besides oxygen, energy and nutrients are the only essentials for human beings to survive. Nutrition is needed constantly to enable all physiological processes to flow smoothly and all organs to function well. If any of more than 30 essential nutrients is lacking for a time, nutrientspecific metabolic pathways and body functions will be

\section{KARGER}

E-Mail karger@karger.com

www.karger.com/ger
(C) 2013 S. Karger AG, Basel

0304-324X/13/0594-0328\$38.00/0
Prof. Dr. Dorothee Volkert

Institute for Biomedicine of Aging

Friedrich-Alexander-Universität Erlangen-Nürnberg

Heimerichstrasse 58, DE-90419 Nürnberg (Germany)

E-Mail dorothee.volkert@iba.fau.de 
impaired and, in the long run, result in characteristic deficiency symptoms. Thus, adequate intake of energy and nutrients is a basic prerequisite for all physiological processes, organ functions, physical and mental performance, health and well-being, and there is no doubt that malnutrition - here defined as sustained intake of energy and/or one or more nutrients below what is required - is a serious phenomenon with far-reaching consequences.

Normally, our natural drive to eat and drink ensures a sufficient intake of food and beverages, allowing an adequate supply of energy and nutrients and resulting in a good nutritional status. With increasing age, this natural drive declines, resulting in the so-called anorexia of aging. A decrease in the sensations of smell and taste, decreasing central orexigenic signals and increasing gastrointestinal satiety signals all contribute to a generally diminished desire to eat in older people [1]. These physiological age-related changes are regarded, on the one hand, as a reasonable adaptation to the natural decrease in energy requirements. On the other hand, they increase the risk of an excessive reduction of food intake and predispose older people to malnutrition. If additional factors like health or social problems accrue, dietary intake is often no longer sufficient to meet requirements.

\section{State of the Art - What Do We Know?}

Since Morley and Silver [2] described for the first time in 1988 the phenomenon of anorexia of aging, much research has been performed which has substantially improved our knowledge in this field.

To begin with, the prevalence of malnutrition has been documented in many studies in different population subgroups confirming malnutrition as a common problem in old people. Recently, in a combined database including 4,507 elderly from different settings in 12 countries, more than two thirds of the participants were found to be at risk of malnutrition (46\%) or to be malnourished (23\%). Whereas malnutrition was found in only $5 \%$ of community-living eldery and 14\% of nursing-home residents, $39 \%$ of geriatric patients in acute-care hospitals and 51\% of patients in geriatric rehabilitation were affected [3]. These results are in accordance with many other studies showing an increasing prevalence of malnutrition with decreasing health and functional status and increasing dependency and disability $[4,5]$. Interestingly, virtually independent of the setting, a large proportion of old people are at risk of malnutrition [3]. Since subjects at risk can be considered likely to develop malnutrition in the

Malnutrition in Older Adults - Urgent Need for Action near future, it is not only malnutrition itself but also the risk of malnutrition which deserves attention.

Secondly, the multifactorial origin of malnutrition is well documented and established. Compared to younger adults, where malnutrition is almost always disease-related, in the elderly, causes are more diverse. Besides the above-mentioned age-related physiological decrease in appetite, many common characteristics of old people including chewing and swallowing problems, physical and cognitive impairment, depression, loneliness and poverty - adversely affect their dietary intake and have repeatedly been shown to be related to malnutrition [4, 6]. In addition, also in the elderly health status is of paramount importance in the etiology of malnutrition. Acute and chronic diseases and concomitant multi-medication may not only reduce appetite and food intake but also increase energy and nutrient requirements, and impair the absorption and alter the metabolism, transport and utilization of nutrients. Moreover, about 15 years ago, acute and chronic inflammation emerged as a key contributing factor in the pathophysiology of disease-related malnutrition [7]. Proinflammatory cytokines compromise nutritional status by reducing appetite, increasing restingenergy expenditure and boosting catabolic processes. The corresponding metabolic syndrome, cachexia, implicates a loss of body weight, mainly muscle mass, aggravating age-related sarcopenia and fueling the vicious cycle of frailty $[8,9]$.

Accordingly, it is important to be aware that body weight and body composition, central characteristics of nutritional status, are not only determined by nutrition but also directly affected by health status. This also applies to several laboratory parameters - albumin, transferrin or lymphocyte count - commonly used for nutritional assessment in previous years. Because these reflect illness rather than true nutritional deficiencies [7], they have retreated into the background of nutritional assessment and made room for malnutrition screening tools which are currently considered state of the art for the identification of subjects with malnutrition or at risk of malnutrition [10]. Considering several aspects of malnutrition simultaneously, screening tools cope better with the complex nature of malnutrition, and are therefore more suitable than single parameters in identifying it. Reduced dietary intake, weight loss, reduced nutritional status (mostly BMI) and disease stress leading to nutritional deterioration are the most important signs of malnutrition and are regarded as essential components of malnutrition screening [10]. Numerous tools have been developed considering these aspects, which are described 
and discussed in detail elsewhere [11-13]. The European Society for Clinical Nutrition and Metabolism (ESPEN) recommends the Malnutrition Universal Screening Tool (MUST) for the community setting, the Nutritional Risk Screening (NRS-2002) for hospitals and the Mini Nutritional Assessment (MNA) for all older people, independent of the setting [10]. The MNA-SF (short-form), specifically developed for the elderly and recently revised and validated [14], in addition to the above-mentioned basic screening parameters, takes into account physical and mental functional impairment, consequently allowing not only the recognition of malnutrition but also of those subjects at risk of malnutrition [15].

The serious consequences of malnutrition have been well documented. Earlier studies focused on clinical outcome and consistently reported an increased risk of morbidity (e.g. the development of pressure ulcers and poor wound-healing, infectious complications, hospital readmissions and increased length of hospital stay) and mortality in malnourished old people $[4,16]$. In recent years, the relation between nutritional deficits and functional impairment has become more and more apparent. It is quite obvious that an energy intake below bodily requirements and the accompanying weight loss aggravate the age-related loss of muscle mass and function. A lack of specific nutrients due to low intake as well as low serum levels have been found to be associated with decreased muscle strength, poor physical performance and disability. The anti-oxidative, anti-inflammatory and anabolic properties of specific nutrients are considered to mediate this relationship [17, 18]. Similarly, due to the role of certain nutrients in brain metabolism and integrity, a lack of these also contributes to cognitive decline; weight loss is also well documented as a common early indicator and attendant symptom of dementia $[19,20]$. Thus, malnutrition has emerged as an important etiological factor in the development of sarcopenia and dementia, two major geriatric health threats, both of which, in turn, provoke nutritional problems and aggravate malnutrition. Particularly in the case of dementia, multiple nutritional problems appear - from anorexia and disturbed eating behavior to dysphagia and a refusal to eat - all compromising adequate dietary intake and worsening nutritional status [20].

In summary, there is no doubt that malnutrition is associated with serious functional and health problems which affect the well-being and quality of life of the individual and also increase costs and burden our health-care system.
Very recently, Morley [21] acknowledged the anorexia of aging and the resultant malnutrition as 'a true geriatric syndrome', characterized by multifactorial causality, identified by a symptom and accompanied by frailty, disability and poor outcome. Thus, malnutrition entered the arena of the 'geriatric giants' and is standing side by side with traditional geriatric syndromes like immobility, instability, incontinence and intellectual impairment.

If we accept nutrition as a basic requirement and malnutrition as a major problem in geriatrics, this should be reflected in everyday patient care. In fact, presently, this seems to not be the case. Despite its frequency and serious consequences, awareness of malnutrition is rather low and prevention and treatment do not receive appropriate attention. Furthermore, shortfalls in nutritional knowledge still have to be complained. For example, in the nutritionDay project in nursing homes, it became apparent that the majority of units performing routine nutritional screening regarded body weight and BMI as screening tools [22]. In an own cross-sectional study in a geriatric acute-care hospital department, we observed a major discrepancy between the actual presence of malnutrition and nutrition-related problems and their identification by physicians and nurses as well as the low percentage of patients actually receiving nutrition support. While malnutrition was found in $25-30 \%$ of the patients according to BMI, Subjective Global Assessment (SGA) and MNA by research staff, the routine documentation in the medical folders revealed a figure of only $6 \%$. Body weight was documented in only half of the patients and BMI was not calculated at all. According to research staff, nutritionrelated problems like loss of appetite or problems with chewing or swallowing were present in up to half of the patients but were also only partly documented by healthcare professionals. Only about $20 \%$ of the malnourished patients received nutritional support [23].

A current postal survey among German geriatric hospital departments also revealed a great potential for improvement in nutritional care. Even though $80 \%$ report routinely documenting body weight and BMI, only $40 \%$ perform a standardized screening for malnutrition with a validated tool. In 13\%, nutritional status is not assessed routinely, and local guidelines for nutritional care are established in only $42 \%$ of the participating hospital units. Ninety percent of the units reported that a speech and language therapist is available in the geriatric department, but only $42 \%$ reported a dietitian or nutritionist being available. In only $15 \%$ of the units, a dietician regularly participates in geriatric team conferences [24]. 
These German data exemplify that malnutrition often remains unrecognized and hence untreated. In many institutions across Europe, neither routine screening for malnutrition nor standards of treatment have been established. Low priority is given to malnutrition in daily routine - despite the fact that effective interventions are available. The beneficial effects of measures to improve the nutritional situation of old people - from nursing and environmental interventions (e.g. eating assistance/feeding, improved mealtime ambience, enough time to eat and emotional support) to the modification of food, meals and snacks, oral nutritional supplements, enteral and parenteral nutrition - are well documented [25-28]. Just recently, it has been shown that comprehensive, individualized nutritional care combining different measures according to individual needs improves the dietary intake, nutritional status, clinical course and quality of life of older patients with malnutrition or at risk of malnutrition [29].

With regard to the correction of malnutrition, it has to be admitted, however, that nutritional interventions are not always (and not to the same extent) successful. Study results on clinical and functional outcomes are less uniform than those on dietary intake and nutritional status, which highlights the complexity of malnutrition and the importance of other factors, namely the type and severity of disease, which also affect these outcomes. Responses to nutritional interventions may be suppressed by inflammation or the progression of a chronic disease, and nutritional efforts may fail, despite thorough consideration and action.

In fact, not every elderly patient showing signs of malnutrition will need and ultimately benefit from nutritional support and, in addition, not everyone will accept nutritional interventions and comply with dietary modifications or promptings to eat.

Therefore, the type and extent of nutritional interventions should be thoroughly reflected in each individual case, and the challenge is often finding the balance between too few and too many. On the one hand, a sufficient intake of energy and nutrients has to be ensured; on the other hand, individual needs and wishes have to be respected and, at the end of life, its natural course, i.e. a decreasing desire to eat, needs to be accepted.

Consequently, knowledge about the complex nature of (mal)nutrition and the above-described interrelations is imperative for adequate nutritional therapy for old people. Without this, in everyday clinical practice, nutritional therapy may be disappointing, which might be one of the reasons explaining its underutilization. Another ex-

Malnutrition in Older Adults - Urgent

Need for Action planation might be the fact that nutritional interventions take time to become effective. In contrast to drugs, which mostly act within hours, it may take several days to weeks until nutritional interventions produce appreciable effects. Most importantly, it has to be acknowledged that nutritional care is not only a matter of supplying energy and nutrients, but also requires individual attention and an affectionate relationship between patient and caregiver. Nutritional interventions are time-consuming, and time is money and is extremely rare in medical and nursing practice nowadays.

\section{Future Prospects - What Do We Need?}

\section{Malnutrition Screening as a Mandatory Part of \\ Geriatric Assessment}

As an important first step towards better awareness, screening for malnutrition should become a mandatory, integral part of the comprehensive geriatric assessment. Malnutrition should be recognized early by routine screening in all health-care settings. As there is no agreed gold standard, an adequate tool has to be selected locally, depending on the setting and practicability. All in all, it is of secondary interest which tool is used, as long as it is validated and contains the essential components described above. The main thing is to screen at all, in order to detect nutritional problems and risk factors for malnutrition as early as possible. A positive screening result will trigger a more detailed nutritional assessment, direct the attention of health-care professionals to nutritional problems and consequently promote nutritional interventions. In the long run, routine consideration of nutritional problems will initiate changes in daily routine and the organization of care, facilitating adequate nutritional support.

Besides routine screening, yearly participation in a benchmark project like the worldwide nutritionDay (www.nutritionday.org) or the International Prevalence Survey of Care Problems (LPZ; www.lpz-um.eu/) may be of value in terms of increasing nutritional awareness.

\section{Implementation of Local Guidelines for Nutritional Care}

Practical local guidelines are also necessary for the improvement of nutritional care in daily routine and should be implemented in all geriatric hospital wards and nursing homes. In recent years, several evidence-based scientific guidelines for nutritional management have been developed at a national and international level dealing with 
medical as well as nursing and catering aspects $[27,28$, $30-32]$. These general, academic guidelines provide the basis for local instructions which should be adapted to the specific situation of each institution and meet the requirements of real life. Old people will only benefit if our huge scientific knowledge is put into practice and is used in daily routine.

\section{Time and Expertise - Adequate Levels of Qualified Staff}

It is important to note that adequate nutritional management will not be possible without adequate staffing levels allowing enough time for individual nutritional care. Regarding their multifactorial origin, nutritional problems - just like other geriatric problems - can only be solved with a multidisciplinary approach. All professional categories involved in geriatric care may also be necessary for the management of malnutrition. In addition, the specific knowledge and experience of a nutritional expert may be required. Consequently, a dietician or nutritionist should be a member of the geriatric team, participate in regular team conferences and contribute to successful holistic patient care.

Last but not least, not only a sufficient number of, but also well-educated staff is needed to ensure the early recognition, effective treatment and - even better - the prevention of malnutrition. Malnutrition will remain a multifaceted phenomenon, requiring basic knowledge on its causes, consequences, diagnosis and therapy as well as a comprehensive understanding of the complex interrelations between malnutrition, aging, functional impairment and disease. Consequently, nutrition has to become part of the basic education and continued training of all health-care professionals, especially medical doctors and nurses. Many health-care professionals involved in geriatrics show great interest in attending specific nutrition training programs [33]. Educational programs would also help private caregivers to learn how to handle nutrihealth require further research. Studies at the cellular, metabolic and clinical levels and linking together information from different research approaches are desirable to expand our still-limited knowledge and to better understand the transition from good nutritional health and independence of old people to malnutrition, functional

needed to develop targeted preventive interventions as well as effective treatment options, and to find the criteria to distinguish those who will benefit from such options from those who will not. There is also still much uncertainty on how to judge the appropriateness of nutritional interventions and the quality of nutritional care.

In parallel to well-designed observational and intervention studies, the standardized documentation of nutritional information in daily routine would not only be helpful for health-care professionals in everyday life to identify health problems and derive care plans, but would also enable the uniform collection of data for research and political decisions. The interRAI for long-term care facilities, for example, recently turned out to be a reliable instrument for the assessment of nursing-home residents of different health systems across Europe [34]. Agreement on a standard definition and diagnostic approach for malnutrition in the elderly among experts - as recently presented for adult malnutrition [35, 36] - would facilitate the attainment of comparable results and draw compatible conclusions.

In summary, nutrition is one important piece of the complex puzzle of aging, health, function and well-being in the elderly and should receive the attention it deserves. The time is ripe for a better inclusion of nutrition in geriatric health care. This will not only bring about improved nutritional status and outcome, and thus individual benefit for the affected patient, but also economic benefits both for the institution and the health-care system [37]. To this end, commitment and relevant decisions are required not only in all geriatric hospital departments and nursing homes but also at the political level. tional problems adequately.

\section{Research and Standardized Data Collection}

In addition to these practical needs, the complex interactions between aging, nutrition, functional status and impairment and poor health. More research is definitely

References
Gerontology 2013;59:328-333 DOI: $10.1159 / 000346142$
1 Di Francesco V, Fantin F, Omizzolo F, Residori L, Bissoli L, Bosello O, Zamboni M: The anorexia of aging. Dig Dis 2007;25:129137.

2 Morley JE, Silver AJ: Anorexia in the elderly. Neurobiol Aging 1988;9:9-16.

3 Kaiser MJ, Bauer JM, Rämsch C, Uter W, Guigoz Y, Cederholm T, Thomas DR, Anthony PS, Charlton KE, Maggio M, Tsai AC, Vellas B, Sieber CC, Mini Nutritional Assessment International Group. Frequency of malnutrition in older adults: a multinational perspective using the Mini Nutritional Assessment. J Am Geriatr Soc 2010;58:17341738.

4 Clarke DM, Wahlqvist ML, Strauss BJG: Undereating and undernutrition in old age: integrating bio-psychosocial aspects. Age Ageing 1998;27:527-534. 
5 Cereda E: Mini Nutritional Assessment. Curr Opin Clin Nutr Metab Care 2012;15: 29-41.

6 McMinn J, Steel C, Bowman A: Investigation and management of unintentional weight loss in older adults. Br Med J 2011;342:d1732.

7 Jensen GL: Inflammation as the key interface of the medical and nutrition universes: a provocative examination of the future of clinical nutrition and medicine. J Parenter Enteral Nutr 2006;30:453-463.

8 Cruz-Jentoft AJ, Baeyens JP, Bauer JM, Boirie Y, Cederholm T, Landi F, Martin FC, Michel JP, Rolland Y, Schneider SM, Topinková E, Vandewoude M, Zamboni M: Sarcopenia: European consensus on definition and diagnosis: Report of the European Working Group on Sarcopenia in Older People. Age Ageing 2010;39:412-423.

9 Jeejeebhoy KN: Malnutrition, fatigue, frailty, vulnerability, sarcopenia and cachexia: overlap of clinical features. Curr Opin Clin Nutr Metab Care 2012;15:213-219.

10 Kondrup J, Allison SP, Elia M, Vellas B, Plauth M: ESPEN guidelines for nutrition screening 2002. Clin Nutr 2003;22:415-421.

11 Bauer JM, Kaiser MJ, Sieber CC: Evaluation of nutritional status in older persons: nutritional screening and assessment. Curr Opin Clin Nutr Metab Care 2010;13:8-13.

12 Donini LM, Savina C, Rosano A, Cannella C: Systematic review of nutritional status evaluation and screening tools in the elderly. J Nutr Health Aging 2007;11:421-432.

13 Green SM, Watson R: Nutritional screening and assessment tools for older adults: literature review. J Adv Nurs 2006;54:477-490.

14 Kaiser MJ, Bauer JM, Ramsch C, Uter W, Guigoz Y, Cederholm T, Thomas DR, Anthony P, Charlton KE, Maggio M, Tsai AC, Grathwohl D, Vellas B, Sieber CC, MNA International Group: Validation of the Mini Nutritional Assessment short-form (MNA$\mathrm{SF})$ : a practical tool for identification of nutritional status. J Nutr Health Aging 2009;13: 782-788.

15 Guigoz Y, Lauque S, Vellas BJ: Identifying the elderly at risk for malnutrition. The Mini Nutritional Assessment. Clin Geriatr Med 2002;18:737-757.

16 Sullivan DH: The role of nutrition in increased morbidity and mortality. Clin Geriatr Med 1995;11:661-672.

17 Milaneschi Y, Tanaka T, Ferrucci L: Nutritional determinants of mobility. Curr Opin Clin Nutr Metab Care 2010;13:625-629.
18 Inzitari M, Doets E, Bartali B, Benetou V, Di Bari M, Visser M, Volpato S, Gambassi G, Topinkova E, De Groot L, Salva A, International Association of Gerontology and Geriatrics (IAGG) Task Force for Nutrition in the Elderly: Nutrition in the age-related disablement process. J Nutr Health Aging 2011;15: 599-604.

19 Solfrizzi V, Panza F, Frisardi V, Seripa D, Logroscino G, Imbimbo BP, Pilotto A: Diet and Alzheimer's disease risk factors or prevention: the current evidence. Expert Rev Neurother 2011;11:677-708.

20 Gillette GS, Abellan VK, Alix E, Andrieu S, Belmin J, Berrut G, et al: IANA (International Academy on Nutrition and Aging) Expert Group: weight loss and Alzheimer's disease. J Nutr Health Aging 2007;11:38-48.

21 Morley JE: Anorexia of aging: a true geriatric syndrome. J Nutr Health Aging 2012;16: 422-425.

22 Valentini L, Schindler K, Schlaffer R, Bucher $\mathrm{H}$, Mouhieddine M, Steininger K, Tripamer J, Handschuh M, Schuh C, Volkert D, Lochs H, Sieber CC, Hiesmayr M: The first nutritionDay in nursing homes: participation may improve malnutrition awareness. Clin Nutr 2009;28:109-116.

23 Volkert D, Saeglitz C, Gueldenzoph H, Sieber CC, Stehle P: Undiagnosed malnutrition and nutrition-related problems in geriatric patients. J Nutr Health Aging 2010;14:387-392.

24 Smoliner C, Volkert D, Wirth R: Management of malnutrition in geriatric hospital units in Germany. Z Gerontol Geriatr 2013; 46:48-55.

25 Hanson LC, Ersek M, Gilliam R, Carey TS: Oral feeding options for people with dementia: a systematic review. J Am Geriatr Soc 2011;59:463-472.

26 Milne AC, Potter J, Vivanti A, Avenell A: Protein and energy supplementation in elderly people at risk from malnutrition. CochraneDatabaseSystRev2009(2):CD003288.

27 Volkert D, Berner YN, Berry E, Cederholm T, Coti BP, Milne A, et al: ESPEN guidelines on enteral nutrition: geriatrics. Clin Nutr 2006;25:330-360.

28 Sobotka L, Schneider SM, Berner YN, Cederholm T, Krznaric Z, Shenkin A, et al: ESPEN guidelines on parenteral nutrition: geriatrics. Clin Nutr 2009;28:461-466.

29 Starke J, Schneider H, Alteheld B, Stehle P, Meier R: Short-term individual nutritional care as part of routine clinical setting improves outcome and quality of life in malnourished medical patients. Clin Nutr 2011; 30:194-201.
30 Thomas DR, Ashmen W, Morley JE, Evans WJ: Nutritional management in long-term care: development of a clinical guideline. Council for Nutritional Strategies in LongTerm Care. J Gerontol A Biol Sci Med Sci 2000;55A:M725-M734.

31 Raynaud-Simon A, Revel-Delhom C, Hébuterne X, French Nutrition and Health Program, French Health High Authority: Clinical practice guidelines from the French Health High Authority: nutritional support strategy in protein-energy malnutrition in the elderly. Clin Nutr 2011;30:312-319.

32 van Asselt DZ, van Bokhorst-de van der Schueren MA, van der Cammen TJ, Disselhorst LG, Janse A, Lonterman-Monasch S, Maas HA, Popescu ME, Schölzel-Dorenbos CJ, Sipers WM, Veldhoven CM, Wijnen HH, Olde Rikkert MG: Assessment and treatment of malnutrition in Dutch geriatric practice: consensus through a modified Delphi study. Age Ageing 2012;41:399-404.

33 Vandewoude M, Michel J-P, Knight P, Anthomy P, Glencorse C, Engfer M: Variablility of nutritional practice by geriatricians across Europe. Eur Geriatr Med 2011;2:67-70.

34 Onder G, Carpenter I, Finne-Soveri H, Gindin J, Frijters D, Henrard JC, Nikolaus T, Topinkova E, Tosato M, Liperoti R, Landi F, Bernabei R: SHELTER project. Assessment of nursing home residents in Europe: the Services and Health for Elderly in Long TERm care (SHELTER) study. BMC Health Serv Res 2012;12:5.

35 Jensen GL, Mirtallo J, Compher C, Dhaliwal R, Forbes A, Grijalba RF, Hardy G, Kondrup J, Labadarios D, Nyulasi I, Castillo Pineda JC, Waitzberg D, International Consensus Guideline Committee: Adult starvation and disease-related malnutrition: a proposal for etiology-based diagnosis in the clinical practice setting from the International Consensus Guideline Committee. Clin Nutr 2010; 29:151-153.

36 White JV, Guenter P, Jensen G, Malone A, Schofield M, Academy Malnutrition Work Group, A.S.P.E.N. Malnutrition Task Force: A.S.P.E.N. Board of Directors Consensus Statement: Academy of Nutrition and Dietetics and American Society for Parenteral and Enteral Nutrition: characteristics recommended for the identification and documentation of adult malnutrition (undernutrition). J Parenter Enteral Nutr 2012;36: 275-283.

37 Elia M: Nutrition and health economics. Nutrition 2006;22:576-578.
Malnutrition in Older Adults - Urgent Need for Action
Gerontology 2013;59:328-333

DOI: $10.1159 / 000346142$ 